\title{
Acute effects of endurance exercise on nocturnal autonomic functions in sedentary subjects: a pilot study
}

\author{
Emi Yuda', Yoshifumi Moriyama², Toshihiko Mori², Yutaka Yoshida', Makoto Kawahara², Junichiro Hayano ${ }^{1, *}$ \\ 'Department of Medical Education, Nagoya City University Graduate School of Medical Sciences, Nagoya, Japan \\ ${ }^{2}$ Kaikoukai Healthcare Group, Nagoya, Japan
}

Nocturnal heart rate variability (HRV) is thought to reflect healthy recovery function of the autonomic nervous system. Although exercise is recommended for health promotion, exercise itself decreases HRV. We studied acute effect of daytime exercise on nocturnal HRV in 5 healthy adults (age, 22-40 years; 2 female subjects) without regular exercise habit. Using a treadmill, they performed 30-min walking at $4 \mathrm{~km} / \mathrm{hr}$ and 30 -min running at $9 \mathrm{~km} / \mathrm{hr}$ from 11 a.m. on different days at an interval of 2 weeks. On these days and a day without exercise (control), Holter electrocardiograms were recorded from 9 a.m. for $24 \mathrm{hr}$. The amplitudes of low-frequency (LF, 0.04-0.15 Hz) and high-frequency (HF, 0.15-0.45 $\mathrm{Hz}$ ) components of HRV were measured continuously by complex demodulation and were averaged over periods of 11:00-11:30 a.m., $3 \mathrm{hr}$ af- ter going to bed, and time in bed at night. Exercise intensities of the walking and running were at $10 \%$ to $44 \%$ and $55 \%$ to $67 \%$ of heart rate reserve, respectively. During exercise, heart rate increased and LF and HF amplitudes decreased with exercise intensity. Nocturnal heart rate and LF and HF amplitude, however, showed no consistent changes with exercise intensity and their averages on the days of walking and running did not differ significantly from those of the control day. In conclusion, 30-min walking and running exercises performed in the morning had no significant acute effects on nocturnal heart rate or HRV.

Keywords: Autonomic nervous system, Complex demodulation, Heart rate variability, Recovery, Sleep

\section{INTRODUCTION}

Since decreased heart rate variability (HRV) was recognized as cardiovascular risk, improvement of HRV has become an important challenge for health science (Task Force of the European Society of Cardiology and the North American Society of Pacing and Electrophysiology, 1996). To increase physical activity has been thought to be a promising approach (Haskell et al., 2007), but studies have reported both positive and negative effects of exercise training on HRV (Boutcher and Stein, 1995; Buchheit et al., 2004; Seals and Chase, 1989; Tulppo et al., 2003). Exercise itself causes acute suppression of HRV (Furlan et al., 1993; Hayano et al., 1994). Thus, the positive effects of daytime exercise may appear only in nocturnal HRV reflecting enhanced autonomic recovery function (Nummela et al., 2016). However, only limited evidence is available for the acute effects of exercise on nocturnal
HRV except for those in athletes (Hautala et al., 2001; Hynynen et al., 2010).

In this pilot study, we examined if nocturnal heart rate and HRV are affected by mild-to-moderate exercise at daytime in subjects without regular exercise habit. In earlier studies of athletes, increased heart rate and decreased HRV during sleep after both 50-min moderate exercise and 3.5-hr marathon run have been reported (Hynynen et al., 2010), while the other study has reported no significant effect of a $75-\mathrm{km}$ cross-country skiing race lasting 4.5 hr on nocturnal absolute HRV (Hautala et al., 2001). Also, in studies of physically active persons, Myllymäki et al. (2012) observed that 30-min mild, moderate, and vigorous intensity endurance exercises starting at 6 p.m. had no significant effect on nocturnal HRV. In these earlier studies, however, the acute effects of exercises on nocturnal HRV might have been confounded by the high level of habitual physical activities and also by the residual
${ }^{\star}$ Corresponding author: Junichiro Hayano (D) https://orcid.org/0000-0002-5340-6325 Department of Medical Education, Nagoya City University Graduate School of Medical Sciences, 1 Kawasumi Mizuho-cho Mizuho-ku, Nagoya 467-8601, Japan Tel +81-52-853-8501, Fax +81-52-853-8761, E-mail: hayano@med.nagoya-cu.ac.jp Received: December 18, 2017 / Accepted: January 20, 2018
This is an Open Access article distributed under the terms of the Creative Commons Attribution Non-Commercial License (http://creativecommons.org/licenses/by-nc/4.0/) which permits unrestricted non-commercial use, distribution, and reproduction in any medium, provided the original work is properly cited. 
effects carried over after the end of exercises (Hautala et al., 2001; Kaikkonen et al., 2007). In the present study, we therefor examined healthy sedentary subjects for the acute effects of exercises performed in the morning.

\section{MATERIALS AND METHODS}

The present study was performed according to the protocol that was approved by the Institutional Review Board of Nagoya City University Graduate School of Medical Sciences and Nagoya City University Hospital (approval number: 60160073).

\section{Subjects}

The subjects were recruited with the following inclusion criteria: healthy nonsmoking persons (a) whose age was between 20 and 40 years, (b) had no regular exercise habit, (c) were not taking medications for $>2$ weeks, and (d) whose electrocardiogram (ECG) displayed a normal sinus rhythm at rest. There were 5 applicants (age: mean \pm standard deviation, $30 \pm 7$ years; range, 22 40 years; 2 female subjects) who met the inclusion criteria and all participated in this study after giving their written informed consent.

\section{Study protocols}

Subjects were instructed to refrain from taking food or beverages containing caffeine or alcohol and from performing strenuous exercise after 7 p.m. the previous night of all of 4 experimental days.

On the first day of experiment, subjects came to laboratory without taking meal and the health conditions including basal metabolic rate, which was measured with a metabolic measurement system (AE-310S, Minato Medical Science Co., Ltd., Osaka, Japan). On the other three experimental days, subjects came to laboratory after taking a light meal for breakfast. Then, Holter ECGs were recorded on these days as control (no exercise), walking, and running exercise days. The recoding was started at 9:00 a.m. and continued for $24 \mathrm{hr}$ including the periods of exercise and sleep. On the control day, subjects spent as usual without exercise. On the walking exercise day, they walked on treadmill (TR33, SPORTSART Industrial Co., Ltd., Tainan, Taiwan) in the laboratory at $4 \mathrm{~km} / \mathrm{hr}$ for $30 \mathrm{~min}$. On running exercise day, they run on the treadmill at $9 \mathrm{~km} / \mathrm{hr}$ for $30 \mathrm{~min}$. On both walking and running exercise days, exercise was started at 11:00 a.m. and ended 11:30 a.m.

\section{Measurements}

The Holter ECG was recorded with a Holter recorder (Cardy 303 pico+, Suzuken Co., Ltd., Nagoya, Japan), by which ECG signals were digitized at $125 \mathrm{~Hz}$ and stored. Digitized ECG data were transferred to a Holter ECG analyzer (Cary Analyzer 05, Suzuken Co. Ltd.), on which all QRS waves were detected and labeled for the rhythm of beat (sinus, ventricular ectopic, supraventricular ectopic, noise, etc.). After all errors in the detection and labelling of QRS waves were edited, R-R interval time series data were obtained. The ratio of sinus-rhythm beats to total beats was calculated for every 1-min segment. The 1-min segments in which the ratio was $<80 \%$ were marked and HRV measures obtained for the segments were excluded from the analyses.

HRV was analyzed by the methods of complex demodulation $(\mathrm{CDM})$ whose detail has been reported elsewhere (Hayano et al., 1993; Hayano et al., 1994). Briefly, CDM is a nonlinear time-domain method of time series analysis that suits to the investigations of nonstationary/unstable oscillations. In contrast to spectral analysis that provides average features (power and frequency) of oscillatory components in stationary state, CDM provides instantaneous amplitude and frequency as functions of time for oscillations in specified frequency band. We used a subroutine CDM written in FORTRAN (the detailed code has been deposited with the National Auxiliary Publications Service) in this study.

To perform CDM analysis, 24-hr R-R interval time series were interpolated only using $\mathrm{R}-\mathrm{R}$ intervals between consecutive $\mathrm{QRS}$ waves in sinus rhythm and resampled at $2 \mathrm{~Hz}$. The time dependent changes in amplitude of low-frequency (LF, 0.04-0.15 Hz) and high-frequency (HF, $0.15-0.45 \mathrm{~Hz}$ ) components were demodulated continuously and LF-to-HF power ratio $(\mathrm{LF} / \mathrm{HF})$ was averaged over sequential 1-min windows (resulting output time resolution $=1 \mathrm{~min}$ ). For the analyses of the LF and HF components, reference frequencies of $0.095 \mathrm{~Hz}$ and $0.30 \mathrm{~Hz}$ were used, respectively. The low pass filtering was performed with a zerophase-shift least-squares filter with convergence factors. The length of the filter was set at 61 terms, resulting in a transitional band width of $0.033 \mathrm{~Hz}$. The low-pass corner frequencies of 0.055 and $0.15 \mathrm{~Hz}$ were used for the LF and HF components so that the demodulating frequency bands for these components were 0.04 to $0.15 \mathrm{~Hz}$ and 0.15 to $0.45 \mathrm{~Hz}$, respectively.

After removing the 1-min segments of data in which the sinus beat ratio was $<80 \%$, LF and $\mathrm{HF}$ amplitudes and $\mathrm{LF} / \mathrm{HF}$ ratio were averaged over periods of 11:00-11:30 a.m., 3 hr after going to bed, and time in bed at night. 


\section{Statistical analysis}

We used SAS 9.1 (SAS Institute Inc., Cary, NC, USA) for statistical analysis. We evaluated data during exercise, 1 st $3 \mathrm{hr}$ after going bed, and time in bed separately. We used the mixed-model analyses of variance for repeated measures with exercise intensity (control, walking, and running), age, and sex as the fixed effects and subject as random effect. $P<0.05$ was considered to be statistically significant.

\section{RESULTS}

Table 1 shows the characteristics of subjects. The exercise inten- sity was presented as $\%$ of heart rate reserve calculated from individual age-dependent estimated maximum heart rate and resting heart rate in each subject. Exercise intensities of the walking and running were at $10 \%$ to $44 \%$ and $55 \%$ to $67 \%$ of heart rate reserve, respectively, and the running had an exercise intensity of $>60 \%$ of heart rate reserve only in subjects 2 and 3 .

Fig. 1 shows heart rate and HRV indices in each subject for each period of the control, walking, and running days. During exercise, HR increased $(P<0.0001)$ and LF and HF amplitudes decreased $(P=0.001$ and $P=0.002)$ with increasing exercise intensity, while LF/HF showed no significant changes. Heart rate, LF and $\mathrm{HF}$ amplitude, and LF/HF during the first $3 \mathrm{hr}$ and whole

Table 1. Characteristics of subjects

\begin{tabular}{|c|c|c|c|c|c|c|c|c|c|c|}
\hline \multirow{2}{*}{ Subject } & \multirow{2}{*}{ Sex } & \multirow{2}{*}{ Age (yr) } & \multirow{2}{*}{ Height (cm) } & \multirow{2}{*}{ Weight (kg) } & \multirow{2}{*}{$\operatorname{BSA}\left(m^{2}\right)$} & \multirow{2}{*}{$\mathrm{BMI}\left(\mathrm{kg} / \mathrm{m}^{2}\right)$} & \multirow{2}{*}{$\begin{array}{l}\text { BEE_Harris } \\
\text { (kcal/day) }\end{array}$} & \multirow{2}{*}{$\mathrm{EE}$ (kcal/day) } & \multicolumn{2}{|c|}{ Exercise intensity $(\%)^{*}$} \\
\hline & & & & & & & & & Walking & Running \\
\hline 1 & Female & 36 & 159.7 & 50.4 & 1.50 & 19.8 & 1,264 & 968 & 28 & 55 \\
\hline 2 & Female & 26 & 164.0 & 65.2 & 1.71 & 24.2 & 1,456 & 1,230 & 28 & 64 \\
\hline 3 & Male & 40 & 166.8 & 74.6 & 1.83 & 26.8 & 1,656 & 1,340 & 43 & 67 \\
\hline 4 & Male & 28 & 172.3 & 76.0 & 1.89 & 25.6 & 1,784 & 1,339 & 28 & 57 \\
\hline 5 & Male & 22 & 168.0 & 56.7 & 1.64 & 20.1 & 1,531 & 1,120 & 10 & 55 \\
\hline
\end{tabular}

$\mathrm{BSA}$, body surface area; $\mathrm{BMI}$, body mass index; $\mathrm{BEE}$, basic energy expenditure; $\mathrm{EE}$, energy expenditure.

${ }^{*}$ Exercise intensity presented as $\%$ of heart rate reserve (age-dependent estimated maximum heart rate - heart rate at rest).

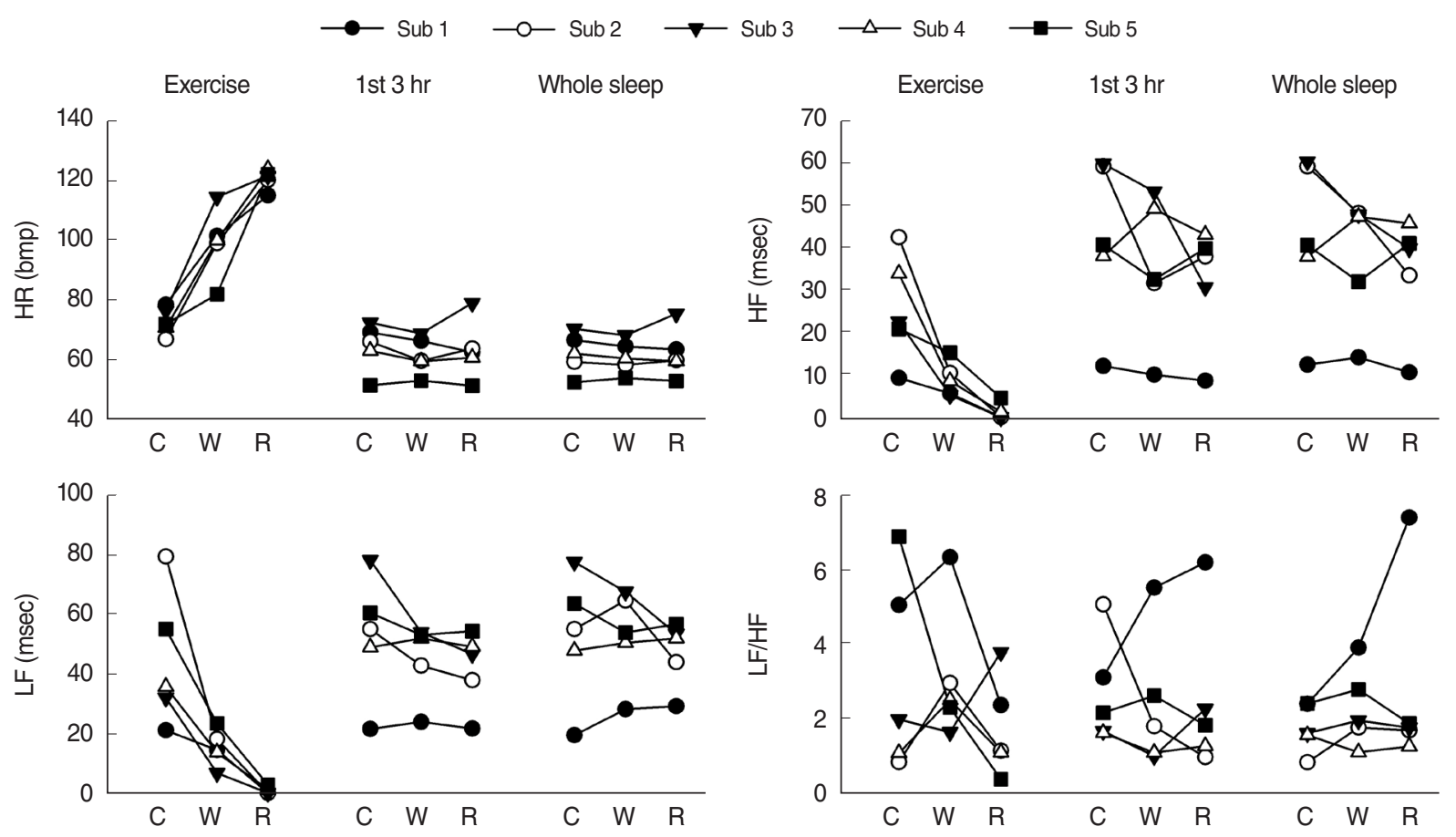

Fig. 1. Effects of walking (W) and running (R) exercises on hear rate (HR) and heart rate variability during exercises and 1st $3 \mathrm{hr}$ and whole sleep periods after exercises compared with data on control (C) nonexercise day. Subjects were 5 healthy sedentary persons (sub 1 to sub 5). LF, low-frequency component amplitude; HF, high-frequency component amplitude; LF/HF, LF-to-HF component power ratio. 
night, however, showed no consistent changes with exercise intensity and did not differ significantly from those of control. Only in subjects 2 and 3, nocturnal LF and HF amplitude on the running day were lower compared with those of control day. In these subjects, the exercise intensity of running was $>60 \%$.

\section{DISCUSSION}

In this pilot study, we examined if nocturnal heart rate and HRV are affected by daytime exercise in subjects without regular exercise habit. We observed that 30-min walking and running exercises performed in the morning had no significant effects on nocturnal heart rate or HRV in these subjects.

Although sleep is an essential recovery function, the information about acute effects of exercise on nocturnal autonomic functions is limited. In an earlier study of 14 healthy male subjects who performed moderate to vigorous intensity physical activities 2 to 3 times a week, Myllymäki et al. (2012) examined the acute effects of mild, moderate, and vigorous intensity 30-min endurance exercises starting at 6 p.m., but they found no significant effect on nocturnal HRV. In a study of 10 healthy male subjects, Hautala et al. (2001) have also reported the acute autonomic effects of a $75-\mathrm{km}$ cross-country skiing race starting in the morning and lasting $4.5 \mathrm{hr}$. They observed increased daytime heart rate and decreased daytime HRV, but no significant changes in nocturnal HRV in absolute values. The results of present study are in the same line with these earlier studies, but our study indicates that the nonsignificant acute effect of exercise on nocturnal HRV is not attributable to high baseline daily physical activities.

It is not possible to determine the mechanisms of our observation in this pilot study, but some speculations for future studies may be possible. There are many studies on the effects of longterm endurance exercise training on HRV (Boutcher and Stein, 1995; Buchheit et al., 2004; Nummela et al., 2016; Seals and Chase, 1989; Tulppo et al., 2003). Although they have reported mixed results, the effects of exercise on nocturnal autonomic function depend on the intensity and duration of exercise training (Nummela et al., 2016). In the present study, the exercise intensity of running estimated by heart rate reserve exceeded $60 \%$ only in subjects 2 and 3, in whom the HF amplitudes during both 1st $3 \mathrm{hr}$ and whole sleep were lower after running than control. Although the basic overload principle of training states that training should be done at a level of load high enough to disturb the homeostasis of the body, the balance between training stress and recovery could be disproportionate. It is unclear whether the acute reduction in HF amplitude is the sign of effective exercise intensity or excessive (harmful) intensity. Future studies deserve to determine if nocturnal HRV could be an indicator of appropriate exercise intensity for safe and effective long-term training for fitness. Particularly, it would be interesting to see the conversion from the initial reduction in nocturnal HRV to the late increase during long-term exercise training processes and to identify the determinants of such processes.

In conclusion, 30-min walking and running exercises performed in the morning had no significant acute effects on nocturnal heart rate or HRV in healthy young sedentary persons.

\section{CONFLICT OF INTEREST}

No potential conflict of interest relevant to this article was reported.

\section{REFERENCES}

Boutcher SH, Stein P. Association between heart rate variability and training response in sedentary middle-aged men. Eur J Appl Physiol Occup Physiol 1995;70:75-80.

Buchheit M, Simon C, Piquard F, Ehrhart J, Brandenberger G. Effects of increased training load on vagal-related indexes of heart rate variability: a novel sleep approach. Am J Physiol Heart Circ Physiol 2004;287: H2813-H2818.

Furlan R, Piazza S, Dell'Orto S, Gentile E, Cerutti S, Pagani M, Malliani A. Early and late effects of exercise and athletic training on neural mechanisms controlling heart rate. Cardiovasc Res 1993;27:482-488.

Haskell WL, Lee IM, Pate RR, Powell KE, Blair SN, Franklin BA, Macera CA, Heath GW, Thompson PD, Bauman A; American College of Sports Medicine; American Heart Association. Physical activity and public health: updated recommendation for adults from the American College of Sports Medicine and the American Heart Association. Circulation 2007;116:1081-1093.

Hautala A, Tulppo MP, Mäkikallio TH, Laukkanen R, Nissilä S, Huikuri HV. Changes in cardiac autonomic regulation after prolonged maximal exercise. Clin Physiol 2001;21:238-245.

Hayano J, Taylor JA, Mukai S, Okada A, Watanabe Y, Takata K, Fujinami T. Assessment of frequency shifts in R-R interval variability and respiration with complex demodulation. J Appl Physiol (1985) 1994;77: 2879-2888.

Hayano J, Taylor JA, Yamada A, Mukai S, Hori R, Asakawa T, Yokoyama K, Watanabe Y, Takata K, Fujinami T. Continuous assessment of hemodynamic control by complex demodulation of cardiovascular vari- 
ability. Am J Physiol 1993;264(4 Pt 2):H1229-H1238.

Hynynen E, Vesterinen V, Rusko H, Nummela A. Effects of moderate and heavy endurance exercise on nocturnal HRV. Int J Sports Med 2010;31: 428-432.

Kaikkonen P, Nummela A, Rusko H. Heart rate variability dynamics during early recovery after different endurance exercises. Eur J Appl Physiol 2007;102:79-86.

Myllymäki T, Rusko H, Syväoja H, Juuti T, Kinnunen ML, Kyröläinen H. Effects of exercise intensity and duration on nocturnal heart rate variability and sleep quality. Eur J Appl Physiol 2012;112:801-809.

Nummela A, Hynynen E, Kaikkonen P, Rusko H. High-intensity endurance training increases nocturnal heart rate variability in sedentary participants. Biol Sport 2016;33:7-13.

Seals DR, Chase PB. Influence of physical training on heart rate variability and baroreflex circulatory control. J Appl Physiol (1985) 1989;66:18861895.

Task Force of the European Society of Cardiology and the North American Society of Pacing and Electrophysiology. Heart rate variability: standards of measurement, physiological interpretation and clinical use. Circulation 1996;93:1043-1065.

Tulppo MP, Hautala AJ, Mäkikallio TH, Laukkanen RT, Nissilä S, Hughson RL, Huikuri HV. Effects of aerobic training on heart rate dynamics in sedentary subjects. J Appl Physiol (1985) 2003;95:364-372. 